\title{
GW23-e1843 REGULATION OF TRANSFORMING GROWTH FACTOR B1 SIGNALLING IN THE POST-ISCHAEMIC MOUSE HEART
}

doi:10.1136/heartjnl-2012-302920a.96

${ }^{1}$ Yuanjing Li, ${ }^{2}$ Guanglong He. ${ }^{1}$ The Department of Cardiology, the First Affiliated Hospital of Chongqing Medical University, Chongqing, China; ${ }^{2}$ Davis Heart and Lung Research Institute and Division of Cardiovascular Medicine, Department of Internal Medicine, Columbus, OH, USA

Objectives eNOS-derived NO induces acute phase tissue hyperoxia in vivo and hyperoxia induces fibroblast trans-differentiation in vitro. However, little is known about the effect of reperfusioninduced hyperoxia on myocardial infarct healing. The current study is to determine how late phase reperfusion hyperoxia and NO regulate cardiac myofibroblast formation.

Methods C57BL/ 6 wild-type, eNOS ${ }^{-/}$and iNOS ${ }^{-/-}$mice were subjected to 30 -min LAD occlusion followed by 14-days of reperfusion. Myocardial tissue $\mathrm{PO}_{2}$ was monitored with electron paramagnetic resonance oximetry. Protein expression of TGF-beta1, p-Smad2/3, t-Smad2/3, p21 and $\alpha$-SMA were measured with ELISA and western blot.

Results There was an acute phase overshoot of tissue $\mathrm{Po}_{2}$ in the WT and $\mathrm{iNOS}^{-/-}$but not $\mathrm{eNOS}^{-/-}$mice. After 60 min reperfusion, tissue hyperoxia was observed in all three groups and peaked at day 3 with significantly lower $\mathrm{PO}_{2}$ in the eNOS ${ }^{-1-}$ mice than that in the WT and $\mathrm{iNOS}^{-/-}$mice $(22.4 \pm 0.8$ vs $39.8 \pm 1.13$ and $26.9 \pm 1.3 \mathrm{~mm} \mathrm{Hg})$. Protein expression of the total and active TGF- $\beta 1, p-S m a d 2 / 3$ over $\mathrm{t}$-Smad2/3 ratio, p21 and $\alpha$-SMA were significantly increased after reperfusion in the WT mice. Knockout of eNOS or iNOS further increased the expression of these signals. Immunohistochemical staining indicated the expression of $\alpha$-SMA in the infarct area. Immunoprecipitation demonstrated the nitration of TGF- $\beta$ RII. Carbogen $\left(95 \% \mathrm{O}_{2}+5 \% \mathrm{CO}_{2}\right)$ treatment increased the expression of $\mathrm{p}-\mathrm{Smad} 2 / 3$ over $\mathrm{t}$-Smad2/3 which was inhibited by EUK134 (10006329 EUK 134) and sodium nitroprusside.

Conclusions Late phase reperfusion tissue hyperoxia promoted while eNOS-/iNOS-derived NO/ONOO- ${ }^{-}$inhibited cardiac TGF- $\beta 1$ signalling and myofibroblast trans-differentiation. These findings may provide new targets to improve myocardial infarct healing and repair. 